\title{
From Research to Reality: Making COSYSMO a trusted estimation tool in your organization
}

\author{
Ricardo Valerdi \\ MIT \\ rvalerdi@mit.edu \\ Chris Miller \\ Systems \& Software Consortium, Inc \\ miller@systemsandsoftware.org \\ Copyright @ 2007 by Ricardo Valerdi and Chris Miller. Published and used by INCOSE with permission.
}

\begin{abstract}
As the COSYSMO model transitions from the development phase into the adoption phase, industry stakeholders are beginning to embrace the model and integrate it into their existing measurement processes. To date, much of the guidance provided by the COSYSMO development team has been focused on the usage of the model. In the adoption phase, users need guidance on how to adopt the model as they work to convince management to invest resources in competition with other process improvement initiatives.

This paper outlines a process which provides guidance on the piloting and institutionalization of COSYSMO designed to help scope the effort needed for successful adoption and implementation. The process has been developed as a result of interactions with over a dozen organizations that have participated in the industry calibration of the model and have begun to integrate the model into their internal processes. The knowledge obtained from working with these organizations is reflected in this process.
\end{abstract}

\section{Experience in Systems Engineering Cost Estimation}

The motivation for this adoption process came from witnessing several organizations struggle with the adoption of credible tools as a result of organizational obstacles. Literature on the topic of technology and innovation adoption points to organizational factors as the main drivers of adoption. A study of technology adoption in the Brazilian medical equipment industry showed that organization factors are the largest contributors to the adoption of an innovation, specifically the presence or absence of leadership supporting the implementation (Zilber et al 2006). Similarly, a study sponsored by the Software Engineering Institute showed that social systems are the main enablers of innovation diffusion in software engineering organizations (Bayer and Melone 1989).

The COSYSMO working group has had an influx of new members. These members lack the historical discussions and background that drove key decisions and assumptions in COSYSMO during its six year development. An organization looking at COSYSMO for the first time may have tacit knowledge of cost modelling in general but may struggle when adopting a new model like COSYSMO. Organizations are jumping on the bandwagon with little or no training and limited knowledge of parameter definition discussions in the absence of any substantial implementation guidance or lessons learned to leverage. In an attempt to help prevent COSYSMO from falling into the silver bullet trap (i.e., "We tried to use COSYSMO but it didn't work"), this paper is aimed at providing just that guidance. By following the systematic 
adoption process outlined in this paper, an organization can maximize the probability of successfully making COSYSMO into a trusted systems engineering estimation capability.

\section{Overview of the Framework}

This framework, shown in Figure 1, provides a systematic approach to increase the probability of successful adoption. The framework involves several steps each described below. The purpose of the framework is to encapsulate the experience and lessons learned during COSYSMO deployments. Certain opportunities exist throughout for verification \& validation of the model and its scope relative to the organization's current measurement system and needs.

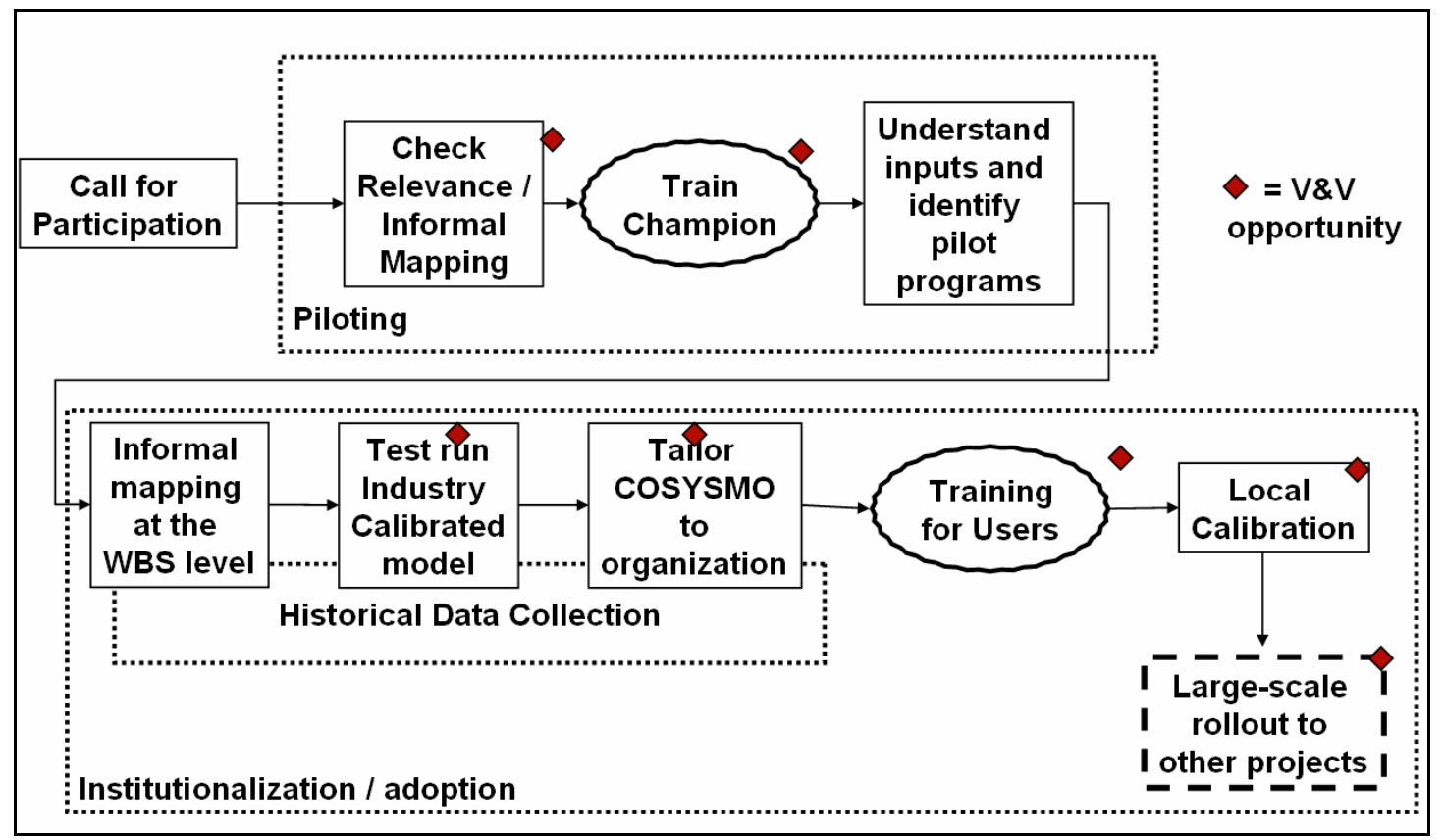

Figure 1. The COSYSMO Adoption Process.

The sequence of steps in the framework are:

1. Call for participation

2. Check relevance/informal mapping

3. Train champion

4. Understand inputs and identify pilot programs

5. Informal mapping at the WBS level

6. Test run industry calibrated model

7. Historical data collection

8. Tailor COSYSMO to organization

9. Training for users

10. Local calibration

11. Large-scale rollout to other projects

It is recommended that organizations review these ten steps and tailor them to maximize the probability of successful adoption of COSYSMO. 
Step 1: Call for participation. The first step involves the solicitation of support and participation in the adoption of COSYSMO. Like any other initiative, implementation of COSYSMO requires significant time and effort before there is a payoff. Identifying a champion and/or sponsor that has the authority to allocate budget and resources to this effort is essential. Our collective experience has shown that trying to implement an estimation tool without management's endorsement will rarely succeed.

Once budget and resources are obtained it is also important to plan this initiative like a project. Using this process and making adjustments for scope (i.e., implementing on a single project or establishing an organization-wide systems engineering estimation capability), is site specific tailoring which needs to be considered. Be sure to capture the desired end state estimation capability. One method is to use prediction intervals (i.e., PRED levels). This simply required the stakeholder to state their expectation for COSYSMO in terms of comparing estimates to actual results. For example, one organization came up with the PRED intervals in Table 1:

Table 1. Organizational PRED Intervals

\begin{tabular}{|l|l|l|}
\hline $\begin{array}{l}\text { Estimates are } \\
\text { within } \boldsymbol{x} \% \text { of actual } \\
\text { Y\% of the time }\end{array}$ & $\begin{array}{l}\text { Current (estimated by } \\
\text { stakeholders via Delphi) }\end{array}$ & $\begin{array}{l}\text { Desired (with } \\
\text { COSYSMO) }\end{array}$ \\
\cline { 2 - 3 }$[$ PRED $(\boldsymbol{x})=\boldsymbol{y}]$ & PRED $(10 \%)=20 \%$ & PRED $(10 \%)=50 \%$ \\
\cline { 2 - 3 } & PRED $(20 \%)=50 \%$ & PRED $(20 \%)=75 \%$ \\
\cline { 2 - 3 } & PRED $(30 \%)=80 \%$ & PRED $(30 \%)=100 \%$ \\
\hline
\end{tabular}

As COSYSMO is implemented, these PRED levels can be used at two major decision points. The first decision point involves determining when COSYSMO is ready to be initially deployed (i.e., sufficient piloting, calibration, and validation). For this decision use the 'current' PRED intervals and only use COSYSMO until its estimation capability has proven to be better, or at a minimum as good, as the current estimation method. The second decision point involves knowing when COSYSMO deployment can be considered complete. Once the established model can be shown to provide estimates as outlined in the 'desired' PRED interval column, then the model implementation phase is complete. For organizations seeking to calculate ROI of COSYSMO, the delta achieved in improving PRED intervals can be converted to cost savings by applying overall project budgets to the reduction in estimation prediction variation.

Most large organizations often have people working on similar issues at different sites. When beginning to evaluate COSYSMO, search out other people within your corporation that may already be using COSYSMO. Connecting with these individuals can help strengthen the case for investing time in COSYSMO and associated activities. Additionally, long term corporate level initiatives may wish to integrate historical data to develop a corporate-wide calibration. Coordination of key model parameters (e.g., definition and collection guidance for collecting size drivers) will help make this feasible.

Step 2: Check relevance/informal mapping. In order to determine the relevance of the model, it is recommended that organizations do an initial feasibility assessment of adopting COSYSMO in their organization. The COSYSMO model makes a few assumptions about the scope of systems engineering activity and the life cycle phases included in the estimate. Reviewing the COSYSMO Users Guide and conducting a high evaluation of COSYSMO will allow an organization to ascertain sufficient knowledge about COSYSMO to determine whether these 
assumptions are compatible with the systems engineering process currently in place. An organization should consider constructing a Day-in-the-Life scenario where they identify the key decision points in their standard project life cycle or in the annual organization operations in which COSYSMO will be leveraged. These scenarios should provide significant insight not only into the feasibility of COSYSMO but also how the model will be integrated with existing processes. The primary objective of this activity is to gain organizational confidence that COSYSMO is a viable technology for the organization. Additionally, this activity will provide greater insight into the inner workings of the COSYSMO model.

Step 3: Train champion. If relevance and applicability have been confirmed, an individual with the appropriate level of influence and visibility should be assigned as the COSYSMO champion. This individual should agree to take on this initiative until it the end, act as the internal advocate, and attend COSYSMO Working Group meetings. From this point forward, the champion will be the central conduit of questions, data collection, analysis, and experience for an organization.

Establishing a champion is a proven best practice. Of the organizations which have a credible software estimation capability, most of them have a champion that embraced the model, took time to understand its functionality, as well as limitations, and personally conducted the care and feeding of the model from its inception to its full instantiation.

The champion will need training from an experienced COSYSMO user on issues related to model inputs, data collection, and calibration. Consider the level of estimation experience, level of systems engineering experience, and familiarity with the project environment (e.g., system domain, acquisition cycle, customer interface, etc.) when selecting an appropriate champion. The ability of the champion to collect historical data, interact with program management, and be recognized as a credible resource on proposal activities plays an important part in successful implementation of an estimation tool.

Step 4: Understand inputs and identify pilot programs. The champion must clearly understand the data requirements for the model inputs and outputs. Most of this information is available through documentation but its use is best learned through tacit knowledge provided by experienced users. This activity refines the Day-in-the-Life scenario by pairing experienced decision makers and program personnel with the champion. In many instances, these experienced individuals also provide pilot programs for the initial roll out of COSYSMO. The purpose of this activity is to walk the experienced personnel through the COSYSMO Data Collection Instrument (DCI) and discuss the model inputs and subsequent output in relation to supporting their decision making. This activity helps the champion better understand the requirements for estimation and it will also help the program personnel understand COSYSMO's strengths and limitations.

The identification of pilot programs is important. The pilot programs represent the way the organization operates and opportunities for tailoring COSYSMO. Be selective in picking pilot projects. Many organizations select pilot programs that are not representative of their typical project, either in size or criticality to the overall business. This creates pilot results that are easily discounted by the rest of the organization when a more significant roll out is attempted. As a practical guide, select pilots that best reflect the organization's standard business, engineering practices, and include typical program and organizational roles. 
Step 5: Informal mapping at the WBS level. At this point in the adoption process, sufficient buy-in should be solidified. It is now important to focus energy on more detailed implementation steps. The first part involves a review of the organization's systems engineering Work Breakdown Structure (WBS). It is important to develop a common effort data collection framework that reflects both COSYSMO and the existing tracking mechanisms of the organization. COSYSMO was developed using a combination of systems engineering standards to create a robust set of effort categories. The champion will need to review the internal company's WBS and time collection structure to customize the model scope. The result is a tailored model that represents the organization's systems engineering activities as reflected in their systems engineering process standard.

The authors have witnessed that many organizations have a less granularity in their existing systems engineering WBS structure than what is provided in COSYSMO. It is important to consider the Day-in-the-Life scenarios when deciding how to best proceed. In many situations, the organization may need to increase the granularity of their effort tracking to obtain data which is required to generate sufficient detail in the model output.

Step 6: Test run industry calibrated model. A parallel activity that can help determine the applicability of COSYSMO is to test the industry calibrated model to determine whether the industry calibrated model estimates systems engineering effort within a reasonable range. Users should be aware that data from the following six organizations was included in the calibration: BAE Systems, General Dynamics, Lockheed Martin, Northrop Grumman, Raytheon, and SAIC. Only these organizations or closely related organizations should interpret the industry calibration of COSYSMO as adequate since it reflects the productivity and business processes inherent in these types of organizations.

Step 7: Historical data collection. The tailored model will provide guidance to revise the data collection instrument to be organization-specific. This data collection instrument is to be used to gather historical information on completed programs. This activity involves the individuals that have access to the quantitative aspects of the project (i.e., size drivers) as well as the stakeholders involved in the project that can assess the qualitative aspects of the project (i.e., cost drivers).

It is not uncommon to have to revisit the parameter definitions during the historical data collection activity. Be prepared to refine the COSYSMO User Guide parameter definitions based on internal engineering process guidance. It is recommended that the champion try to align the data collection instrument with the standard processes and procedures guidance.

Step 8: Tailor COSYSMO to organization. If any customization was previously identified in terms of the model definitions, scope, and activities, it should be implemented in this step. The primary purpose of this activity is to take the knowledge obtained from the previous steps and reorient the guidance to be reflective of the organization. Some groups take the opportunity to rename their tool at this step because refining the model input parameters, effort categories, and coupling the model output with key decision points significantly converts the academic COSYSMO model into an organization-specific estimation model.

This step also provides an opportunity to revisit the model requirements and create any supplemental material that may be needed prior to beginning the next several steps which involve rolling the model out to the organization. 
Step 9: Training for users. As the model becomes ready for use, users will need to be trained. Creating sufficient training to expose them to the model inputs to use the model is essential. Training programs which require extensive labor are likely to be abandoned due to cost and time constraints. Insufficient training may leave users confused when they attempt to the model and create frustration that will drive them away from using the tool. Deliberate consideration should be invested into how best train to train the target user group, but 1-day courses seem to work well in our experience.

In addition to receiving training on how to develop systems engineering effort estimates, users should also be exposed to the main assumptions of the model and the local tailoring that has been performed as a result of the organization's dominant processes.

Step 10: Local calibration. Once a critical mass (i.e., at least 5 data points) of historical data has been obtained, it can be used to derive a local calibration of COSYSMO. This is an essential step for improving accuracy and relevance of the model. Based on the availability of pilot programs, the authors recommend using COSYSMO in a support capacity until the 'current' estimation capability PRED intervals are surpassed, as described in Step 1. At which time, there is objective evidence that COSYSMO provides more accurate estimates than the current estimation method.

Step 11: Large-scale rollout to other projects. Armed with a locally calibrated model, the COSYSMO champion can field a large scale implementation of the model that reaches across the organization. There are many non-technical considerations associated with such a roll out. For example, rogue program managers may simply refuse to trust a parametric model over their experience and judgment. Many barriers to full scale implementation can be avoided if some initial planning takes into account probable issues (i.e., risks) based on change managerial habits within their organization.

Ultimately, COSYSMO should be used in parallel with other estimation approaches. The difference between the estimates from COSYSMO and other methods can provide useful information about the unique characteristics of the program being estimated.

\section{Next Steps}

In the short term, the COSYSMO working group is continuing to refine the input parameter definitions and model usage guidance. The adoption process outlined in this paper is being used and refined based on inputs from organizations active in implementation. The authors plan to incorporate lessons learned into Chapter 2 of the upcoming COSYSMO book.

In the longer term, the authors will integrate other industry systems engineering measurement activities (e.g., LAI SE Leading Indicators Guide, INCOSE Measurement Working Group Guidance, and Practical Software and Systems Measurement) with COSYSMO. The end goal is to provide continual guidance on systems engineering estimation to industrial affiliates in order to help them adopt the COSYSMO model successfully. 


\section{References}

Bayer, J., Melone, N., A Critique of Diffusion Theory as a Managerial Framework for Understanding Adoption of Software Engineering Innovations, Journal of Systems and Software, Vol. 9, 1989.

Valerdi, R., Raj, J., "Sea Level Requirements as Systems Engineering Size Metrics," $15^{\text {th }}$ INCOSE Symposium, July 2005, Rochester, NY.

Valerdi, R., Miller, C., Thomas, G., “Systems Engineering Cost Estimation by Consensus,” $17^{\text {th }}$ International Conference on Systems Engineering, September 2004, Las Vegas, NV.

Valerdi, R., Rieff, J., Roedler, G., Wheaton, M., "Lessons Learned for Collecting Systems Engineering Data," $2^{\text {nd }}$ Conference on Systems Engineering Research, April 2004, Los Angeles, CA.

Valerdi, R., Boehm, B., Reifer, D., "COSYSMO: A Constructive Systems Engineering Cost Model Coming Age,” $13^{\text {th }}$ INCOSE Symposium, July 2003, Crystal City, VA.

Zilber, M. A., Cosentino, H. M., Lex, S., Factores de Organización y Adopción de un a Innovación: Un Estudio Explorador de la Industria Brasileña de Equipamientos Electromédicos, Journal of Technology Management \& Innovation, Vol. 1, No. 5, 2006.

\section{BIOGRAPHIES}

Ricardo Valerdi is a Research Associate at MIT in the Lean Aerospace Initiative and the developer of COSYSMO. He obtained his M.S. and Ph.D. from the University of Southern California and his B.S. from the University of San Diego.

Chris Miller is the Chief Technologist in Measurement at SSCI and the Chair of the INCOSE Measurement Working Group. He is a Ph.D. candidate at George Washington University. He obtained his B.S. from Penn State University. 